\title{
Early outcome after implantation of bioresorbable vascular scaffolds in elderly patients
}

\author{
Authors: Amr Abdin, Mohammed Saad and Martin Hoher
}

\begin{abstract}
Aims
To evaluate the incidence of MACE in patients who are more than 70 years old and treated with bioresorbable vascular scaffold (BVS).

\section{Methods}

We retrospectively analysed the data of 152 patients who presented to our department and were treated with percutaneous coronary intervention (PCI) using BVS. A total of 58 patients of more than 70 years of age (group A) were compared with 94 patients younger than 70 years (group B). We analysed the data during hospital stay and at 3-6 months regarding the occurrence of MACE (death, myocardial infarction and culprit vessel revascularisation). Angiographic follow-up was done after 3-6 months for all patients.
\end{abstract}

\section{Results}

Procedural success was obtained in all patients at the time of intervention. During hospital stay, there was one death in each group ( $1.72 \%$ in group A vs $1.06 \%$ in group $B, p=0.619$ ). Mortality in group A was probably not related to the scaffold, as the patient died one day after PCI because of cardiogenic shock following rupture of the anterior myocardial wall. The difference in BVS thrombosis was insignificant between the two groups ( $1.72 \%$ in group A vs $3.19 \%$ in group $\mathrm{B}, \mathrm{p}=0.506)$. There was only one case of BVS restenosis in group B. The cumulative incidence of MACE showed no significant difference between the two groups treated with the BVS (3.45\% in group A vs $4.26 \%$ in group $\mathrm{B}, \mathrm{p}=0.583$ ).

\section{Conclusions}

Elderly patients treated with BVS did not show higher rates of MACE compared with younger patients treated with BVS at 3-6 months follow-up.

\section{Conflict of interest statement}

We don't have any conflicts of interest. 